Case

\title{
Aorto-right Ventricular Fistula: A Complication of Aortic Valve Replacement
}

\author{
Mohammad Q. Najib, MD, Daniel Ng, MD, Karyne L. Vinales, MD, and Hari P. Chaliki, MD
}

\begin{abstract}
The occurrence of aorto-right ventricular (aorto-RV) fistula after prosthetic aortic valve replacement is rare. Transthoracic echocardiography (TTE) with color-flow Doppler, transesophageal echocardiography (TEE), or both may be required for diagnosis. A 42-yearold woman sought care for palpitations and dyspnea due to atrial flutter 2 weeks after prosthetic aortic valve replacement and graft replacement of the ascending aorta. TTE and TEE revealed left-to-right shunt due to aorto-RV fistula.
\end{abstract}

Keywords: aortic valve surgery, aorto-right ventricular fistula, echocardiography, transesophageal echocardiography

\section{Introduction}

The most common cardiac complications after prosthetic valve replacement include prosthetic valve endocarditis, thromboembolism, pericardial effusion, hemolysis, and valve failure resulting in prosthetic or periprosthetic regurgitation and/or obstruction. ${ }^{1)}$ The occurrence of aorto-right ventricular (aorto-RV) fistula after prosthetic aortic valve replacement, however, is rare. We describe the case of a 42-year-old woman in whom aorto$\mathrm{RV}$ fistula developed after aortic prosthetic valve replacement.

\section{Case Report}

A 42-year-old woman sought care at the emergency department for acute onset of palpitations and dyspnea. Her medical history was significant for bicuspid aortic valve and ascending aortic aneurysm. Fifteen days earlier,

Division of Cardiovascular Diseases, Mayo Clinic, Scottsdale, Arizona, USA

Received: January 19, 2011; Accepted: March 23, 2011

Corresponding author: Hari P. Chaliki, MD. Division of Cardiovascular Diseases, Mayo Clinic, 13400 E Shea Blvd, Scottsdale, AZ 85259, USA

Email: chaliki.hari@mayo.edu

(C)2011 The Editorial Committee of Annals of Thoracic and Cardiovascular Surgery. All rights reserved. the patient had undergone aortic valve replacement with a 23-mm prosthesis (St Jude Medical, St Paul, Minnesota) in the intra-annular position, using mattress sutures of 2-0 Ethibond (Ethicon, Somerville, New Jersey) with felt pledgets. At the same time, excision and graft replacement of the ascending aorta was performed with a $28-\mathrm{mm}$ synthetic vascular graft (Hemashield; Boston Scientific Corporation, Natick, Massachusetts). Her early postoperative course was excellent with no recurrence of symptoms. On initial evaluation in the emergency department, she had atrial flutter with a rapid ventricular rate of 150 beats per minute. A complete blood cell count and serum electrolyte levels were within normal limits. There was no evidence of infective endocarditis. Her international normalized ratio was subtherapeutic at 1.2. Chest radiography showed an increased cardiothoracic ratio with normal pulmonary vascularity.

Transesophageal echocardiography (TEE) after anticoagulation with unfractionated heparin was performed to rule out intracardiac thrombus in preparation for direct-current cardioversion. TEE images did not show any thrombus in the left atrial appendage, entire left atrium, and the pulmonary veins. Mechanical aortic valve function was normal. However, the TEE short-axis view of the aortic valve showed a continuous color-flow jet at the aortic annulus level into the right ventricle (RV) (Fig. 1A and 1B) (Movie). Additional images from both TEE and transthoracic echocardiography (TTE) 

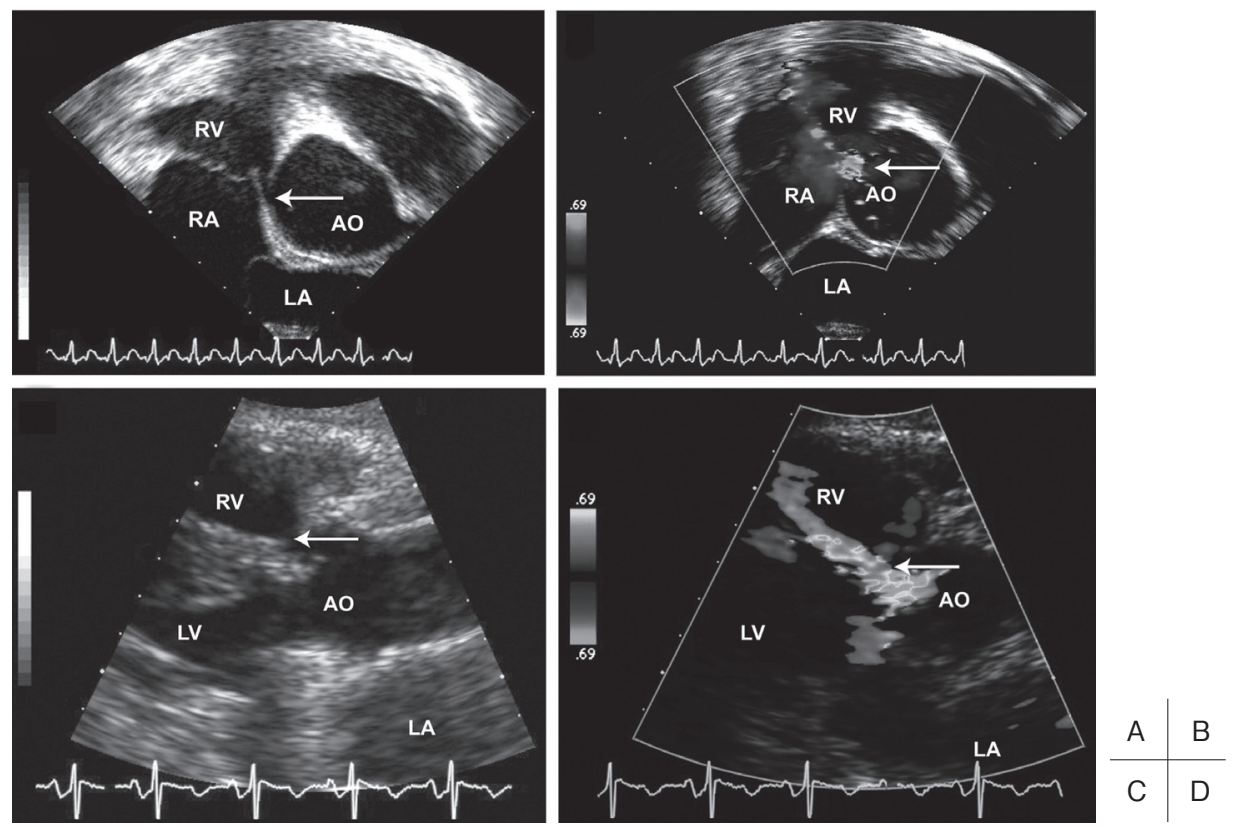

Fig. 1 Two-Dimensional Echocardiographic Views. A and B, Transesophageal echocardiographic short-axis views of the aorta (AO) without (A) and with (B) color-flow Doppler imaging. A communication was seen (arrow) from the aorta to the right ventricle (RV). The flow enters the RV below the tricuspid valve septal leaflet (Movie). C and D, Transthoracic parasternal long-axis views showing aorto-RV fistula (arrow) without (C) and with (D) color-flow Doppler imaging. Communication was seen between the aorta and RV near the right aortic sinus (C), with the flow entering from the aorta into the RV (D).

LA: left atrium; LV: left ventricle; RA: right atrium

confirmed an abnormal communication (of $3.1 \mathrm{~mm}$ ) between the right aortic coronary sinus and the RV (Fig. 1C and 1D). RV size and systolic pressure $(21 \mathrm{~mm}$ $\mathrm{Hg}$ ) were normal. A continuous-wave Doppler study demonstrated unidirectional flow from the aorta to the RV.

The presence of high velocities $(>3 \mathrm{~m} / \mathrm{s})$ throughout the cardiac cycle, due to a significant pressure gradient in systole and diastole (Fig. 2), was consistent with aorto$\mathrm{RV}$ fistula. In contrast, in a left ventricle-to-RV fistula, most of the flow would be seen only during systole because of the minimal gradient between the left and right ventricles during diastole. The ascending aortic graft beyond the sinotubular junction appeared intact. The patient was discharged from the hospital in normal sinus rhythm after a successful cardioversion. The shunt volume was believed to be small, given the normal RV size and systolic pressure. Endocarditis prophylaxis and cardiac follow-up were recommended to the patient. During 9 months of postoperative follow-up, she had no recurrence of symptoms. TTE performed 31 months after her surgery showed no change in the aorto-RV fistula,

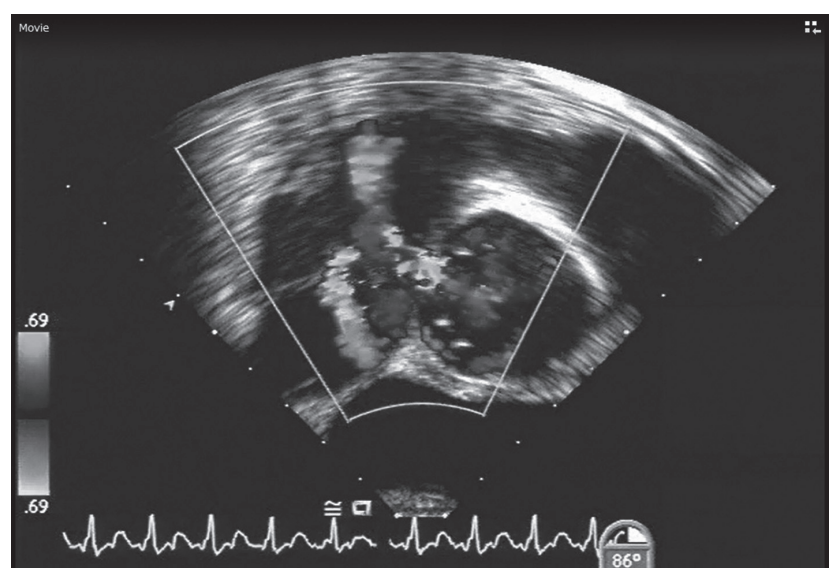

\section{Supplementary Movie}

This supplementary material is available online (supplement to Figure 1).

Transesophageal echocardiographic short-axis view of aorta with color-flow Doppler. Two-dimensional images show a communication from the aorta to the right ventricle. The flow enters the right ventricle below the tricuspid valve septal leaflet. 


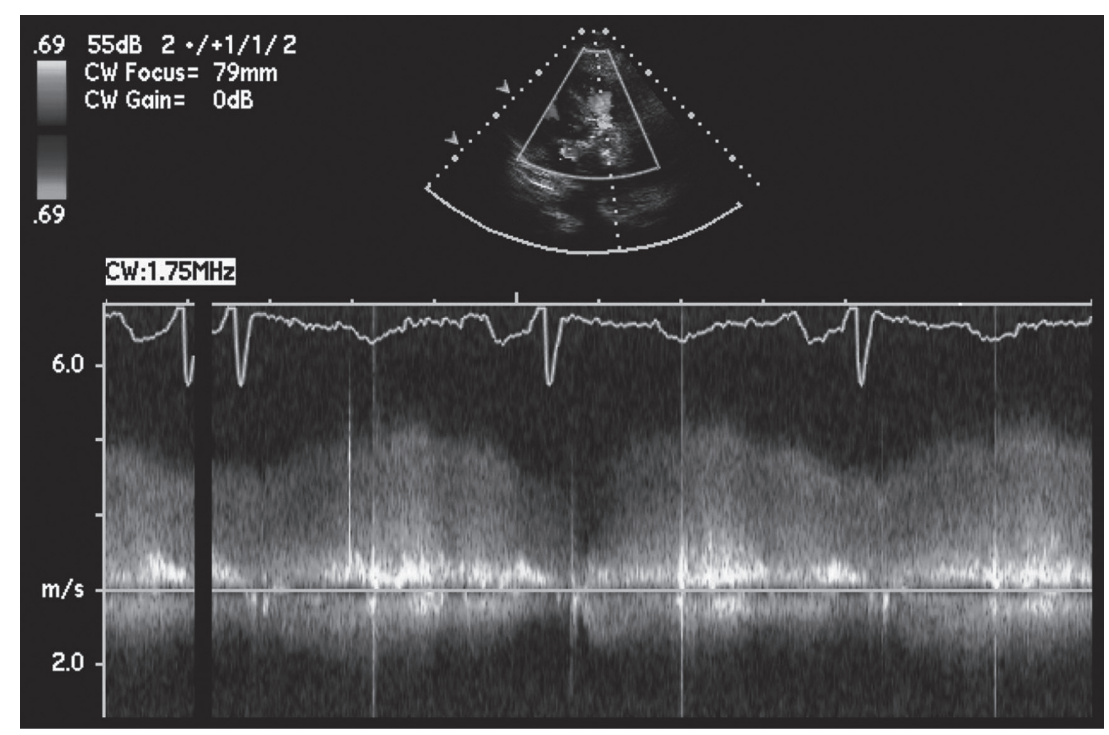

Fig. 2 Doppler Echocardiography. Continuous-wave Doppler tracing of flow from the aorta to the right ventricle. Tracings depict continuous flow through the fistula consistent with aorto-right ventricular fistula.

with normal right-sided heart size and function and normal estimated pulmonary artery systolic pressure.

\section{Discussion}

In general, aorto-RV fistulas have been described to occur because of rupture of congenital and acquired sinus of Valsalva aneurysms. Although rare, blunt trauma to the chest, penetrating chest trauma, aortic dissection, high ventricular septal defect repair, ${ }^{2)}$ and aortic valve surgery ${ }^{2-5)}$ have been reported to result in aorto-RV fistula. The true incidence of aorto-RV fistula after aortic valve surgery is unknown. However, Jackson et al. ${ }^{5)}$ reviewed a series of 310 patients with aortic valve replacement and found only 1 case of fistula from the aorta to the right atrium as well as the $\mathrm{RV}$, suggesting that the incidence most likely is less than $0.5 \%$.

Several mechanisms of injury causing cardiac shunts during aortic valve surgery have been proposed, including inadvertent injury to the membranous septum during dissection below the noncoronary cusps, perivalvular damage attributable to improper retraction and excessive debridement of calcium from the annulus, ${ }^{2,4)}$ and pseudoaneurysm formation secondary to degenerative changes at the aortotomy line and the pseudoaneurysm's eventual rupture into the $\mathrm{RV}{ }^{1)}$ Lorenz et al. ${ }^{2)}$ proposed that another cause may be inclusion of the membranous portion of the ventricular septum in the prosthetic valve suture line, causing ischemic necrosis and eventual development of
aorto-RV fistula. Aorto-RV fistula after prosthetic aortic valve replacement secondary to the trauma sustained during surgery has also been reported. ${ }^{3)}$

The clinical presentation of aorto-RV fistula after aortic valve surgery depends on the size of the shunt. An aorto-RV fistula causes a continuous murmur, ${ }^{6}$ a thrill ${ }^{3,5,6)}$ or both. ${ }^{3,5)}$ Patients with a small aorto-RV fistula may be completely asymptomatic and may have an associated murmur only. ${ }^{2,3)}$

The natural history of aorto-RV fistula after aortic valve surgery has not been well studied. However, the natural history of traumatic aorto-RV fistula was reported by Samuels et al. ${ }^{7}$ In their series of 40 patients, symptoms of heart failure developed over variable intervals, and definitive surgical repair of the aorto-RV fistula was performed in 38 of 40 patients. The mean interval between the time of injury and definitive repair was 1.5 years, with a satisfactory surgical outcome in all patients. Many patients in their series (35\%) had concomitant aortic valve regurgitation; thus, it is not clear whether surgery was needed as a result of the aortic valve regurgitation or the large left-to-right shunt caused by trauma.

Nevertheless, spontaneous closure of aorto-RV fistula has not been reported, and thus, careful follow-up of all patients with aorto-RV fistula is prudent. ${ }^{6,8)}$ We recommend yearly follow-up of patients with small shunts, paying particular attention to right heart chamber dimensions and pulmonary artery systolic pressure. With advances in interventional cardiology, it is now possible to close the 
aorto-RV fistula percutaneously using devices such as a septal occluder (Amplatzer device; AGA Medical Corporation, Plymouth, Minnesota) with 3-dimensional TEE guidance in patients who are poor surgical candidates. ${ }^{4)}$

\section{Conclusion}

The presentation of aorto-RV fistula after prosthetic aortic valve replacement is an exceedingly rare condition. TTE with color-flow Doppler, TEE, or both may be required for diagnosis. Careful follow-up of these patients is essential because heart failure may develop in some patients, necessitating surgical correction of the fistula.

\section{References}

1) Roy D, Saba S, Grinberg I, et al. Aorto-right ventricular fistula: a late complication of aortic valve replacement. Tex Heart Inst J 1999; 26: 140-2.

2) Lorenz J, Reddy CV, Khan R, et al. Aortico-right ventricular shunt following aortic valve replacement.
Chest 1983; 83: 922-5.

3) Chow WH, Lee PK, Cheung KL, et al. Two-dimensional and pulsed Doppler echocardiographic diagnosis of an acquired aortic right ventricular fistula. Clin Cardiol 1989; 12: 544-5.

4) Eng MH, Garcia JA, Hansgen A, et al. Percutaneous closure of a para-prosthetic aorto-right ventricular fistula. Int J Cardiol 2007; 118: e31-4. Epub 2007 Mar 26.

5) Jackson DH Jr, Murphy GW, Stewart S, et al. Delayed appearance of left-to-right shunt following aortic valvular replacement. Report of two cases. Chest 1979; 75: $184-6$.

6) Cakir C, Duygu H, Kilicaslan B, et al. Postoperative diagnosis of aorto-right ventricular outflow tract fistula caused by stab wound: a case report. J Am Soc Echocardiogr 2007; 20: 1415.e5-7. Epub 2007 Jul 12.

7) Samuels LE, Kaufman MS, Rodriguez-Vega J, et al. Diagnosis and management of traumatic aorto-right ventricular fistulas. Ann Thorac Surg 1998; 65: 288-92.

8) Fierro-Renoy C, Pastor-Cervantes JA, Anderson M, et al. Aorto-right ventricular outflow tract fistula: presentation 1 year after stab wound to the heart. Echocardiography 2001; 18: 183-4. 